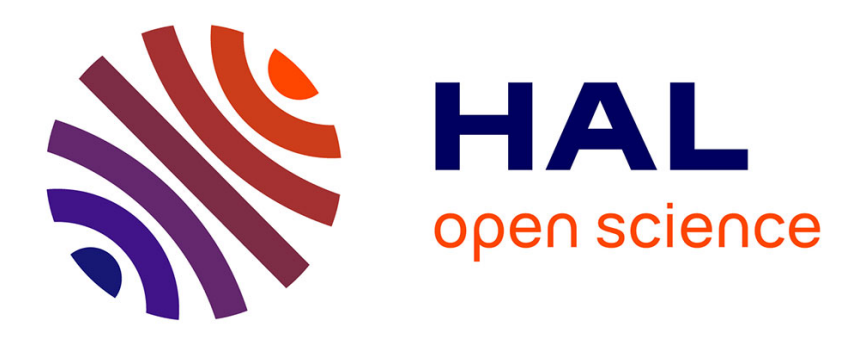

\title{
Bien-être et comportement chez les oiseaux domestiques
} J.M. Faure, A.D. Mills

\section{To cite this version:}

J.M. Faure, A.D. Mills. Bien-être et comportement chez les oiseaux domestiques. Productions Animales, 1995, 8 (1), pp.57-67. hal-00896104

\section{HAL Id: hal-00896104 https://hal.science/hal-00896104}

Submitted on 1 Jan 1995

HAL is a multi-disciplinary open access archive for the deposit and dissemination of scientific research documents, whether they are published or not. The documents may come from teaching and research institutions in France or abroad, or from public or private research centers.
L'archive ouverte pluridisciplinaire HAL, est destinée au dépôt et à la diffusion de documents scientifiques de niveau recherche, publiés ou non, émanant des établissements d'enseignement et de recherche français ou étrangers, des laboratoires publics ou privés. 
INRA Prod. Anim., $1995,8(1), 57-67$
J.M. FAURE, A.D. MILLS

INRA Station de Recherches Avicoles 37380 Nouzilly

\section{Bien-être et comportement chez les oiseaux domestiques}

Les problèmes de bien-être sont importants pour au moins deux raisons : par leur impact auprès du public, ils peuvent déconsidérer une production aux yeux des consommateurs ; par leurs conséquences sur la productivité qu'ils peuvent diminuer à travers les phénomènes de stress.

Cet article présente deux approches des problèmes de bien-être : l'adaptation de l'environnement à l'animal et l'adaptation de l'animal à l'environnement. Ces deux méthodes ne doivent pas être vues comme concurrentes mais au contraire comme complémentaires. Elles ne peuvent cependant permettre de résoudre que les conséquences de l'absence de bien-être sur la productivité, car l'impact auprès du public est en grande partie non rationnel.

\begin{abstract}
A l'origine, les problèmes de bien-être ont été posés par les groupes de protection des animaux, principalement de l'Europe du nord, en raison de l'industrialisation de l'élevage qui leur a paru se faire au détriment des animaux. Leur démarche inclut une forte proportion d'anthropomorphisme ( Aimeriez-
\end{abstract}

\section{Résumé}

La notion de bien-être recouvre deux aspects : la perception par le public (consommateurs) des conditions dans lesquelles sont élevés les animaux domestiques et la perception par l'animal des conditions dans lesquelles il est élevé.

Seul ce deuxième aspect relève de l'approche éthologique. Deux voies sont utilisables pour améliorer le bien-être des animaux : l'adaptation de l'environnement à l'animal et l'adaptation de l'animal à l'environnement.

Dans le premier cas le préalable indispensable est la connaissance des besoins de l'animal, ce qui, même chez la poule, est loin d'être acquis. Quelques exemples sont donnés à partir de quatre méthodes d'approche différentes : comparaison des comportements dans différents milieux, conditionnement opérant, test de choix et conflit de motivation.

L'adaptation de l'animal à l'environnement peut se faire par voie ontogénétique ou génétique et deux exemples sont donnés.

Les deux voies d'approche doivent être utilisées en parallèle. vous vivre dans les conditions faites aux animaux domestiques?") ou de passéisme selon laquelle l'élevage traditionnel est bon, l'élevage industriel mauvais. C'est pour cette raison que les élevages extensifs trouvent facilement grâce à leurs yeux malgré les problèmes de parasitisme ou de conditions climatiques extrêmes qu'ils peuvent poser. De la même façon, la truie à l'attache est perçue comme subissant un traitement cruel alors que la vache à l'attache ne pose aucun problème à la plupart des protecteurs des animaux.

De nombreuses définitions du bien-être ont été proposées mais beaucoup d'entre elles ne présentent qu'une vue partielle du problème. On peut dire que le bien-être d'un animal est satisfaisant s'il se sent en sécurité (s'adapte avec succès et contrôle son environnement), n'éprouve pas de douleur (blessure ou maladie) et ne présente pas de symptôme d'ennui ou de frustration.

Deux voies sont possibles pour améliorer le bien-être des animaux :

- On peut essayer d'adapter l'environnement à l'animal, mais les environnements proposés en élevage industriel ont été développés en raison de leur efficacité. Tout chan- 
gement a donc un coût économique et la décision ne peut être prise qu'en étant sûr de connaître réellement les besoins de l'animal, et donc que le changement proposé correspondra bien à une amélioration de son bien-être.

- On peut aussi adapter l'animal à l'environnement. Plusieurs voies d'approche sont possibles : chirurgie, pharmacologie, ontogenèse, génétique. Cependant les méthodes chirurgicales (débéquage, écornage) ou pharmacologiques (calmants) sont également très mal perçues par les protectionnistes et par les consommateurs et leur utilisation tend à être limitée par la loi. Nous ne nous intéresserons donc qu'aux deux autres voies d'approche. Les méthodes tendant à améliorer le bien-être par voie ontogénétique ou génétique ont aussi un coût. La sélection en particulier n'est utilisable que si le caractère sélectionné est suffisamment général pour apporter une amélioration quel que soit le milieu dans lequel est élevé l'animal. Sinon, un changement de mode d'élevage peut réduire à néant le résultat d'une sélection qui, même chez les espèces à intervalle de génération court, représente un travail de plusieurs années.

\section{1 / Adapter l'environnement à l'animal}

\section{1 / Connaître les besoins de l'animal}

Le préalable à cette démarche est de connaître les besoins de l'animal, définis comme les ressources pour lesquelles la demande de l'animal est inélastique (au sens micro-économique) par opposition à une préférence pour laquelle la demande est élastique (c'est-à-dire diminue quand la difficulté rencontrée pour l'obtenir augmente). Plusieurs voies d'approche sont possibles et nous en examinerons quatre.

\section{a / Comparaison des comportements exprimés dans différents milieux}

Cette méthode n'est applicable que si les milieux diffèrent par un nombre réduit de paramètres, ou mieux, un seul. Dans tous les autres cas on observe de très nombreuses différences de comportement mais leur interprétation est difficile.

Un bon exemple de ce type de travail est extrait de la thèse de Nicol (1987). Cet auteur a étudié, chez la poule pondeuse, l'évolution de seize mesures de comportement dans des cages de hauteur variable. Sur les seize mesures, seules six présentaient des différences significatives en fonction de la hauteur de la cage et ont été représentées sur la figure 1.

On constate que pour les comportements de type toilette (se secoue, étire la tête, se gratte la tête) il y a augmentation de la fréquence de
Figure 1. Fréquence de certains comportements en fonction de la hauteur de la cage chez les poules pondeuses (d'après Nicol 1987).

fréquence des comportements
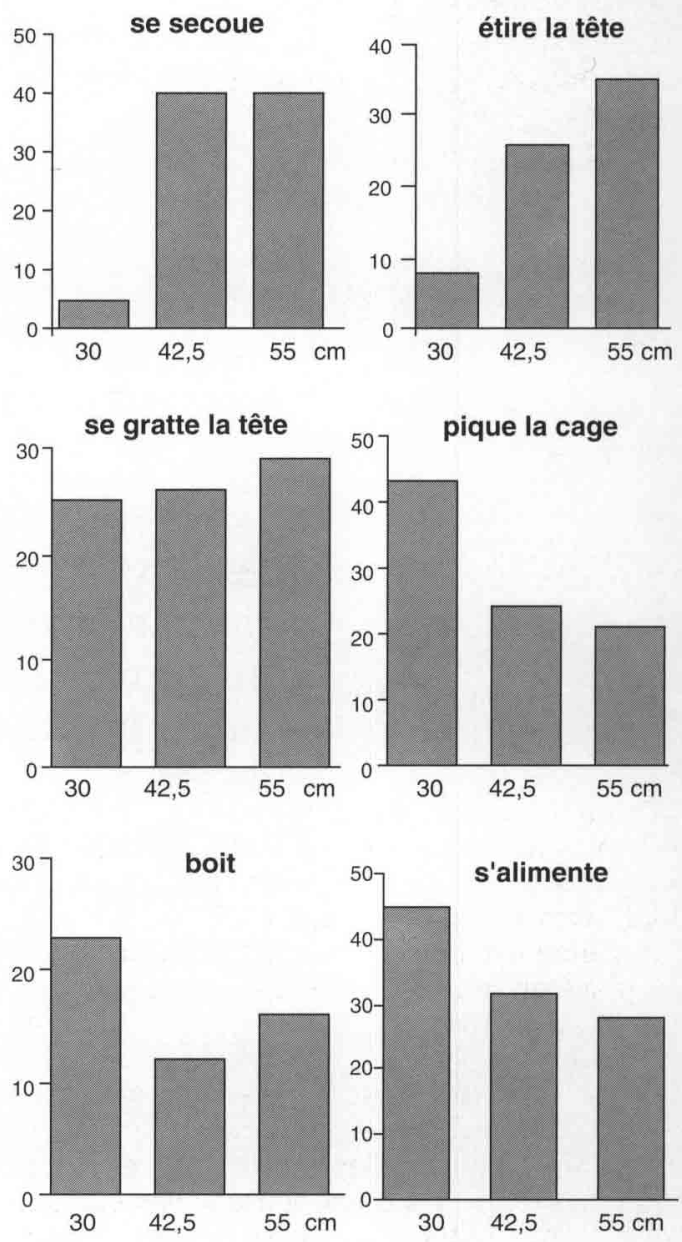

Hauteur de la cage

ces comportements avec l'augmentation de la hauteur de la cage. Au contraire, pour les comportements de type alimentaires (pique la cage, boit, s'alimente), il y a diminution de la fréquence liée à la diminution de la hauteur de cage. Pour la plupart des critères les différences sont importantes entre la hauteur $30 \mathrm{~cm}$ et les 2 autres valeurs testées. Par contre, il y a peu de différence entre $42,5 \mathrm{~cm}$ et $55 \mathrm{~cm}$ de hauteur.

Ces résultats montrent qu'une augmentation de la hauteur de la cage de $30 \mathrm{~cm}$ à $42,5 \mathrm{~cm}$ améliore le bien-être des poules mais que l'augmentation de $42,5 \mathrm{~cm}$ à $55 \mathrm{~cm}$ n'entraîne pas d'amélioration supplémentaire.

\section{b / Conditionnement opérant}

La méthode du conditionnement opérant consiste à apprendre à un animal à effectuer un acte (appuyer sur un bouton par exemple) pour obtenir une récompense ou éviter une punition. 
Figure 2. Schéma des cages de conditionnement pour l'espace (a) et pour la mangeoire (b).

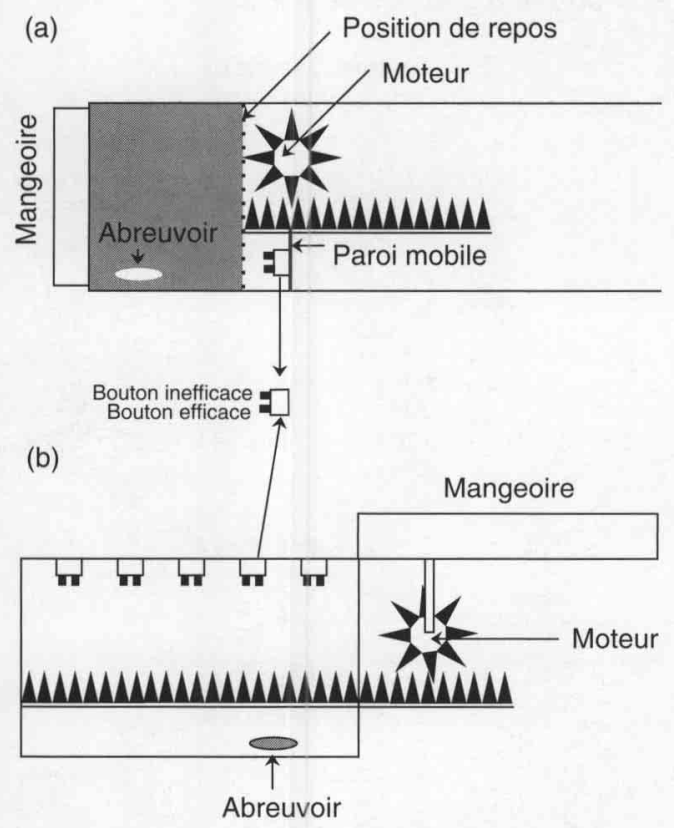

Nous avons adapté cette méthode à l'étude des besoins en espace chez la poule. Pour cela les poules disposent d'une cage (figure 2a) dont trois des parois sont fixes alors que la quatrième est mobile. Sur cette paroi mobile est placé un groupe de deux boutons dont l'un est efficace (un appui sur ce bouton entraîne un déplacement de la paroi mobile sur une distance et pendant un temps fixé par l'expérimentateur) alors que l'autre est inefficace (ne provoque pas de déplacement de la paroi). L'appui sur un des deux boutons est enregistré par l'ordinateur qui pilote la cage. L'enregistrement comprend l'identité du bouton et l'heure de l'appui (à la seconde près). Après appui sur le bouton efficace il peut y avoir ou

Figure 3. Influence de la taille de la récompense sur le travail effectué (nombre d'appuis) en conditionnement opérant pour l'accès à la mangeoire ou à plus d'espace. La faible récompense correspond à un déplacement de $10 \mathrm{~cm}$ ou de $5 \mathrm{~cm}$, la forte récompense de 20 ou de $10 \mathrm{~cm}$ pour la mangeoire et la paroi respectivement.

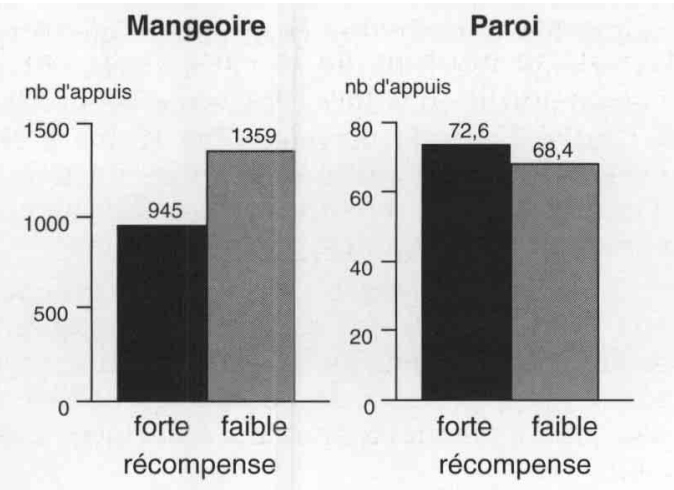

non retour à une position de repos pré-établie. La surface de la cage peut varier de $1600 \mathrm{~cm}^{2}$ à $6100 \mathrm{~cm}^{2}$ et les animaux sont toujours testés en groupes de 4 poules pendant des périodes de plusieurs jours consécutifs (cage paroi).

Un dispositif similaire (figure $2 \mathrm{~b}$ ) permet de mesurer le travail effectué par les poules pour agrandir leur mangeoire de 0 (mangeoire non accessible) à $100 \mathrm{~cm}$ (cage mangeoire).

La présence d'un bouton inefficace permet de vérifier que les appuis sur les deux boutons ne se font pas au hasard. Dans ces conditions expérimentales il est possible d'appliquer la théorie de la consommation (Dawkins 1983) qui prévoit que si la récompense correspond à un besoin, la diminution de la taille de la récompense (longueur de déplacement de la paroi ou de la mangeoire) entraîne une augmentation du travail (nombre d'appuis sur le bouton).

La figure 3 montre que c'est bien le cas dans la situation de la cage mangeoire puisqu'il y a une forte augmentation du nombre d'appuis. Par contre, dans la situation de la cage paroi, il n'y a pas d'augmentation du nombre d'appuis. Une plus grande surface ne correspond donc pas à un besoin pour les poules.

On peut cependant se poser la question de savoir si la poule est capable de faire le lien entre l'appui sur un bouton et la variation de l'espace disponible (Dawkins et Beardsley 1986). On observe bien que les poules, même dans le cas du conditionnement pour l'espace, utilisent plus le bouton efficace (près de $75 \%$ des appuis) que le bouton inefficace mais ce fait est insuffisant pour être sûr que les appuis sont bien effectués pour agrandir la cage.

On peut programmer le système de façon à obtenir cinq conditions expérimentales différentes:

a) Agrandissement de la cage à partir d'une surface de repos de $1600 \mathrm{~cm}^{2}$.

b) Agrandissement de la cage à partir d'une surface de repos de $2500 \mathrm{~cm}^{2}$.

c) Diminution de la cage à partir d'une surface de repos de $2500 \mathrm{~cm}^{2}$.

$\mathrm{d}$ et e) Idem b et c, mais à partir d'une surface de repos de $4300 \mathrm{~cm}^{2}$.

On constate (figure 4) que les poules travaillent pour agrandir une petite cage ( 1600 ou $2500 \mathrm{~cm}^{2}$ ) mais pas pour agrandir une grande cage $\left(4300 \mathrm{~cm}^{2}\right)$ ou pour diminuer sa surface. Dans les 3 derniers cas il y a extinction du conditionnement.

Ce résultat montre que les poules sont capables :

- d'estimer la taille de leur cage et d'adapter leur réponse à cette taille;

- d'apprécier le sens du déplacement puisqu'elles continuent à appuyer sur le bouton pour agrandir mais pas pour diminuer une cage de $2500 \mathrm{~cm}^{2}$.

\section{La taille de la récompense a un effet sur le travail que fournit la poule pour avoir accès à la mangeoire mais pas pour l'accès à un espace plus grand.}


Figure 4. Travail effectué (variation de la surface de la cage) avec 3 surfaces de repos différentes par des poules travaillant pour augmenter (barres vers le haut) ou pour diminuer la taille de leur cage. Les barres ayant des lettres différentes (a ou b) représentent des valeurs significativement différentes.

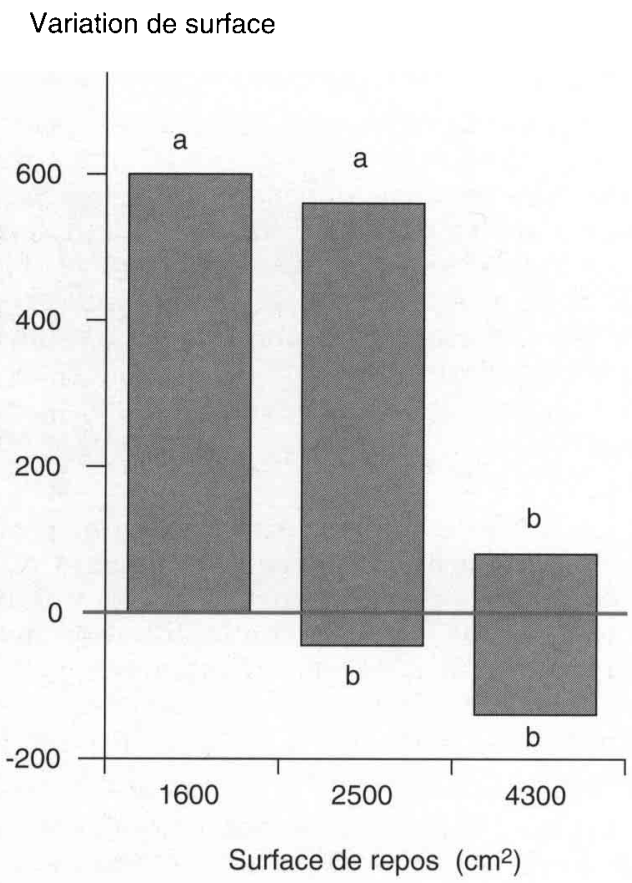

\section{c / Test de choix}

L'appareil utilisé pour le conditionnement opérant peut aussi être utilisé pour un test de choix. Dans ce cas le retour automatique à une position de repos est supprimé et l'un des deux boutons permet d'agrandir la cage alors que l'autre bouton permet de diminuer sa surface. Huit groupes ont été testés dans ce dispositif pendant 12 jours chacun. Ces 12 jours ont été divisés en 3 périodes de 4 jours

Figure 6. Dispositif permettant d'envoyer un vent de vitesse variable sur la mangoire. Trois des 4 couloirs de distribution du vent sont représentés fermés (noir) et un ouvert (blanc à gauche).
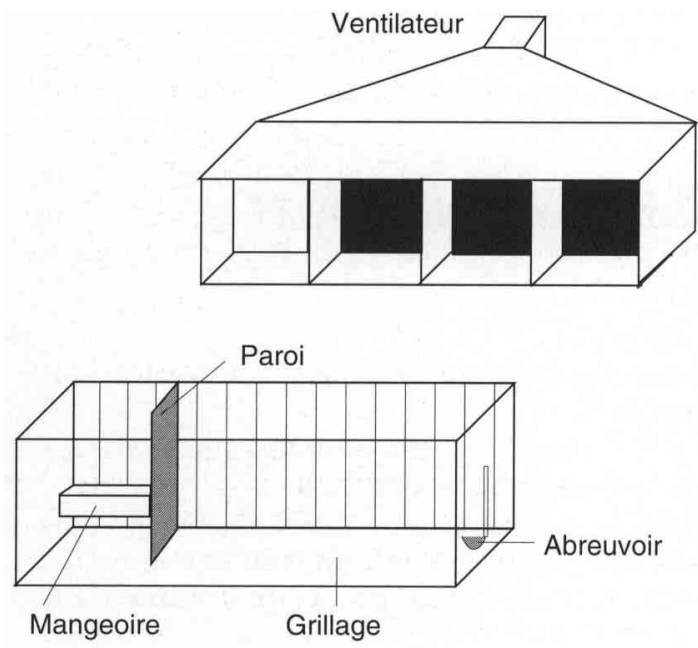

Figure 5. Distribution du temps d'accès aux différentes surfaces possibles de cage en situation de choix par 8 groupes de poules.
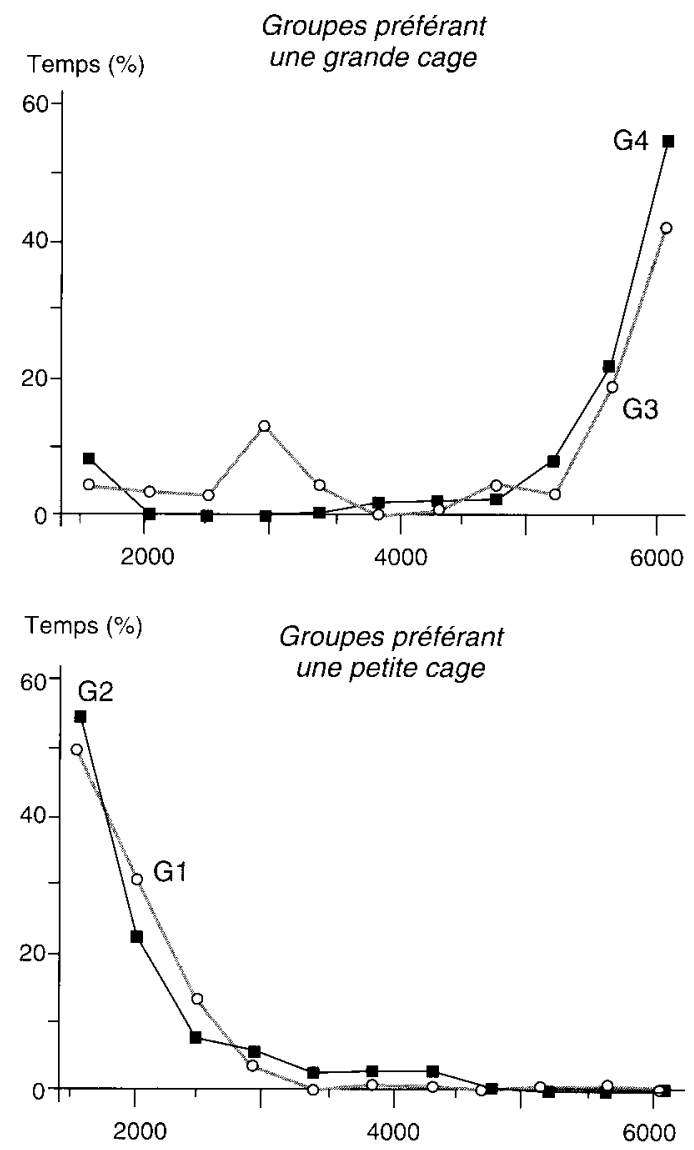

Temps (\%)

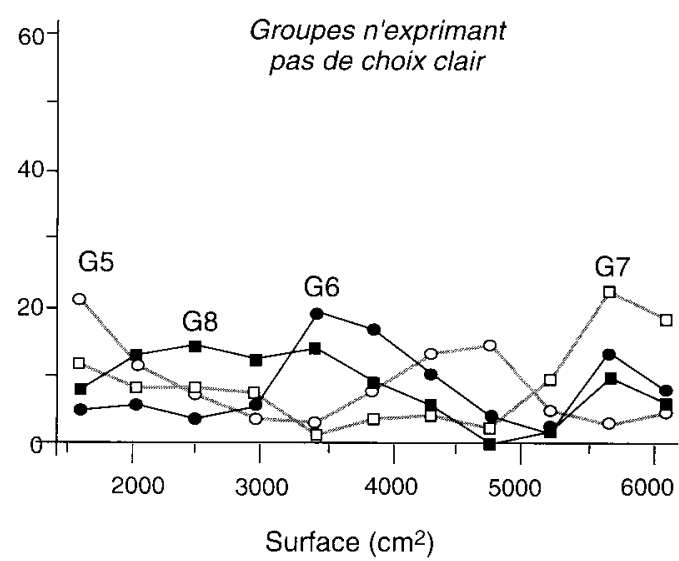

et au début de chaque période de 4 jours la position de départ de la paroi était fixée de manière à obtenir une cage petite, moyenne ou grande, ces 3 situations étant présentées dans un ordre aléatoire différent d'un groupe à l'autre. Seule la moyenne des 12 jours est présentée car la taille de la cage au début d'une période de 4 jours n'a pas d'influence sur sa surface moyenne.

On constate (figure 5) que deux des groupes ont toujours choisi une grande cage, 2 groupes ont toujours choisi une petite cage alors que 4 groupes de poules ont utilisé, à peu près également, toutes les surfaces possibles. 
Les préférences des poules varient donc d'un groupe à l'autre et 6 groupes sur 8 préfèrent, au moins à certains moments, une cage plus grande que celle qui leur est habituellement offerte.

\section{d / Conflit de motivation}

Dans cette situation expérimentale, l'animal peut accéder à un élément de l'environ-

Figure 7. Elasticité (pente de la droite de régression) de la demande pour l'aliment après 0 , 12 ou 24 heures de privation alimentaire face à des vents de 0 à $6 \mathrm{~m} / \mathrm{s}$. Le temps d'alimentation est représenté en pourcentage du temps passé en libre accès pour chacune des durées de privation alimentaire.

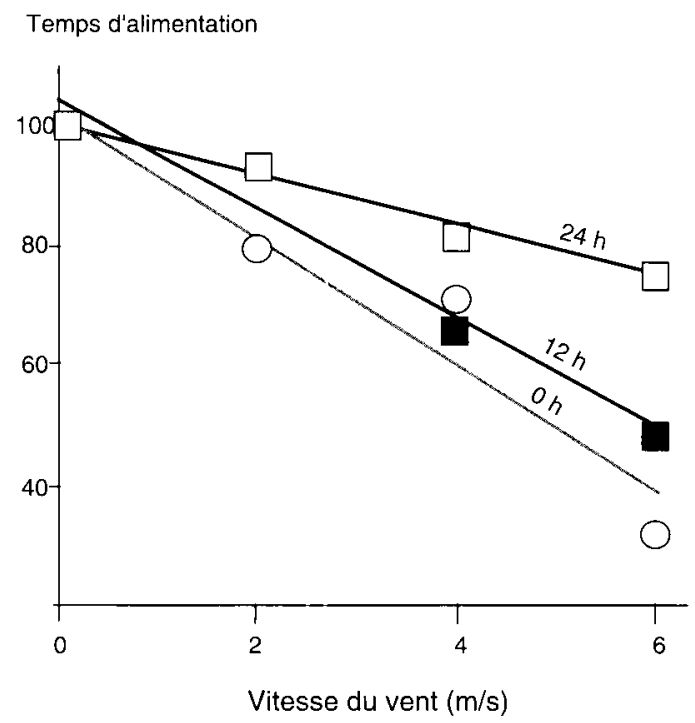

$$
\begin{array}{ll}
0 \mathrm{~h}-\mathrm{y}=102,40-10,80 x & \mathrm{r}^{2}=0,932 \\
12 \mathrm{~h}-\mathrm{y}=103,80-9,10 x & \mathrm{r}^{2}=0,966 \\
24 \mathrm{~h}-\mathrm{y}=100,10-4,45 \mathrm{x} & \mathrm{r}^{2}=0,993
\end{array}
$$

Figure 8. Dispositif permettant d'envoyer un vent de vitesse variable sur une zone de la cage recouverte de sable ou de grillage.

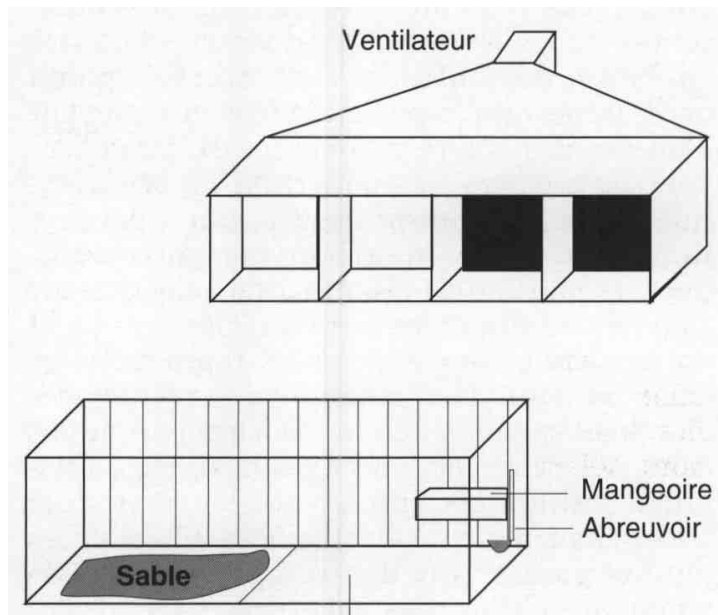

Grillage nement supposé attractif à condition d'accepter en même temps de subir une stimulation déplaisante. Une stimulation relativement répulsive pour les poules et qui est aussi facilement variable est le vent. Comme pour le conditionnement, on peut appliquer dans ce cas la théorie de la consommation, puisque si l'élément attractif correspond à un besoin, la réponse doit être inélastique (le temps passé à consommer l'élément attractif ne varie pas avec la vitesse $d u$ vent).

Nous avons testé la validité de la théorie à l'aide du dispositif illustré par la figure 6. Les résultats obtenus montrent que si on augmente la motivation alimentaire (par privation) on diminue la pente de la droite de régression et donc l'élasticité (figure 7 ). La théorie est donc bien confirmée.

Un des besoins supposé des poules est l'accès à une surface dans laquelle elles peuvent gratter, picorer ou faire des nids. Nous avons donc comparé (figure 8) le temps passé par des poules, soumises à un courant d'air, sur une surface recouverte de grillage ou de sable. La figure 9 montre que les poules ont tendance à préférer le sable (elles y passent plus de temps) mais que l'élasticité observée pour le sable et pour le grillage ne sont pas différentes. Le sable ne correspond donc pas à un besoin.

\section{2 / Applications possibles de ces études}

Quand les besoins des animaux sont connus, on peut essayer d'y répondre en modifiant les conditions d'entretien. Ces conditions ont toutefois été fixées en prenant principalement en compte les facteurs économiques et ceci est

Figure 9. Elasticité (pente de la droite de régression) de la demande pour un accès à du sable ou à du grillage face à des vents de 0 à $3 \mathrm{~m} / \mathrm{s}$. Le temps de présence est représenté en pourcentage du temps passé en libre accès pour les deux conditions expérimentales.

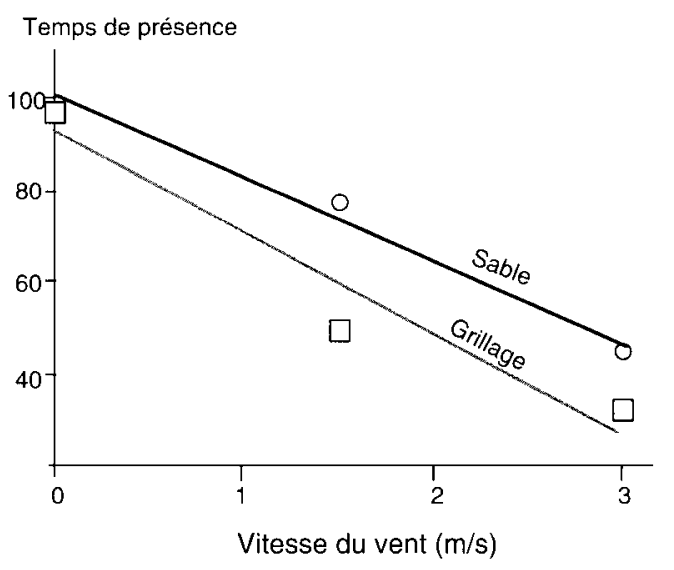

Sable: $\quad y=102,000-18,667 x$

$r^{2}=0,985$

Grillage : $y=94,500-23,000 x$

$r^{2}=0,929$
Le temps passé dans des conditions désagréables varie peu avec

l'intensité de la stimulation dans le cas d'un besoin, par exemple alimentaire pour un animal affamé, alors qu'il varie beaucoup s'il ne s'agit pas d'un besoin, par exemple présence de sable ou aliment pour un animal non affamé. 
particulièrement vrai dans le cas des élevages industriels. Les conditions d'élevage sont donc généralement optimales sur le plan économique et tout changement de ces conditions aura un coût. Il est donc d'autant plus important de disposer de méthodes permettant de hiérarchiser les besoins afin de satisfaire préférentiellement ceux qui sont prioritaires pour les animaux. Il faut aussi noter que l'amélioration des conditions d'élevage est une décision qui est du domaine politique puisqu'il faut mettre en balance, d'une part le bien-être des animaux, et d'autre part le coût qui devra être supporté par le producteur ou par le consommateur. Dans ce domaine, la Commission des Communautés Européennes et le Conseil de l'Europe ont un rôle leader. Leurs décisions manquent cependant souvent de bases scientifiques et le dernier projet de la CEE (projet Mac Sharry) qui ferait passer la surface minimale par poule de $450 \mathrm{~cm}^{2}$ à $800 \mathrm{~cm}^{2}$ et la hauteur de la cage de 40 à $60 \mathrm{~cm}$, multiplie en fait par 2,7 le volume occupé par une poule. Le coût en est donc très important alors que, comme nous l'avons vu précédemment, la hauteur de la cage et la surface disponible ne sont pas des paramètres essentiels pour les animaux, au moins dans les normes actuellement utilisées.

\section{2 / Adapter l'animal à l'environnement}

Cette adaptation peut être réalisée de plusieurs façons : voie chirurgicale, pharmacologique, ontogénétique ou génétique. Les voies pharmacologiques et chirurgicales ne seront pas discutées plus avant car elles posent, soit des problèmes de bien-être (cas du débecquage), soit des problèmes de résidus, très mal perçus par le consommateur (voie pharmacologique).

\section{1 / Ontogénèse}

Certains comportements sont très fortement influencés par les conditions d'élevage précoce. C'est en particulier le cas des relations homme-animal chez le poussin (Jones et Faure 1981) ou chez la vache (Boissy et Bouissou 1988) et du comportement de perchage chez la poule (Faure et Jones 1982). Dans le cas du poussin, il a été constaté que la manipulation des animaux (transférés deux fois par jour de leur cage d'élevage à une boîte, puis de la boîte à leur cage) suffisait à réduire la peur de l'homme: les poussins manipulés présentent une réaction d'immobilité tonique plus courte et des comportements d'approche de l'expérimentateur plus marqués que les témoins. Par contre leurs réactions de peur sont identiques dans les tests n'impliquant pas une composante humaine importante (test d'open-field et d'approche d'un objet nouveau).

Dans le cas du comportement de perchage, on constate que des animaux habitués à se
Figure 10. Temps de perchage dans trois souches de poules alors que les animaux sont âgés de 18 semaines quand ils disposent de l'accès au perchoir depuis une (a) ou 14 semaines (b).

Temps de perchage

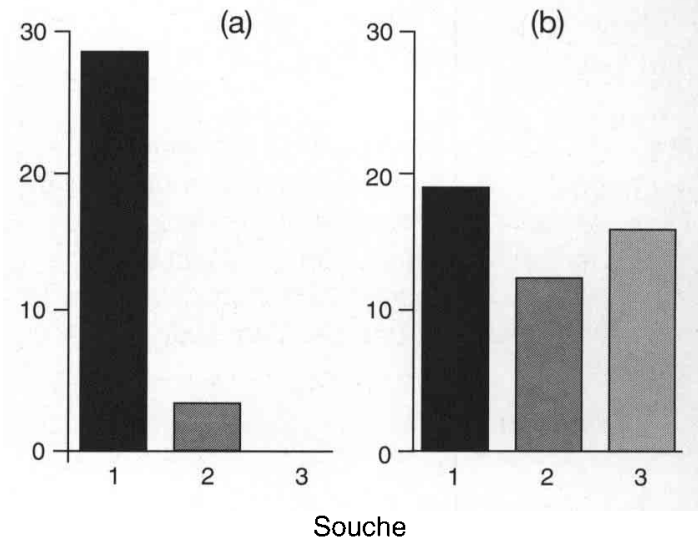

percher seulement à l'âge adulte le font tout à fait normalement dans certaines souches alors que d'autres ne se perchent que peu ou pas du tout (figure 10).

Au contraire, si les animaux ont accès très tôt (à l'âge de 4 semaines) à des perchoirs, toutes les souches étudiées se perchent avec des fréquences comparables. Ce résultat montre que certaines souches de poules ne peuvent apprendre à se percher que si elles ont eu accès très tôt à des perchoirs et montre donc une interaction génotype $\mathrm{x}$ environnement.

Ces deux exemples mettent en évidence la possibilité de modifier les comportements ultérieurs de l'animal par des interventions sur les conditions d'élevage du jeune animal.

\section{2 / Problèmes de génétique du comportement}

Les caractères de comportement sont généralement difficiles à sélectionner, non pas parce qu'ils présentent des héritabilités différentes de celles des autres caractères, mais en raison des difficultés rencontrées quand on veut mesurer rapidement le comportement d'un grand nombre d'animaux. Il est cependant de plus en plus important de sélectionner les animaux domestiques, et en particulier les oiseaux, pour leur comportement, dans la mesure où le milieu de sélection est souvent différent du milieu d'élevage. C'est en particulier le cas pour les reproductrices chair ou leurs descendants qui sont toujours élevés au sol alors que les souches parentales sont sélectionnées en cages-batteries. Jusqu'au milieu des années 60 , les reproductrices chair étaient sélectionnées au sol et les poules présentant des comportements de ponte au sol (hors des nids trappe) ou de couvaison étaient éliminées. Depuis que la sélec- 
tion se fait en cage, les animaux susceptibles de présenter ces comportements ne sont plus éliminés, puisque le milieu de sélection ne permet pas leur expression, et on rencontre de plus en plus de problèmes de ponte au sol ou de couvaison en élevage de production.

On peut sélectionner des animaux bien adaptés à un environnement donné ou des animaux très adaptables. La deuxième solution semble de loin la meilleure puisque les conditions d'élevage ont largement évolué au cours des dernières décennies et qu'il est probable qu'elles évolueront encore.

La sélection est un processus relativement lent et la sélection d'animaux trop spécialisés pourrait être inefficace si, pendant le processus de sélection, les conditions d'élevage ont évolué de manière trop importante.

\section{3 / Génétique : l'exemple de la sélection des cailles}

Une sélection des cailles pour leur comportement a été entreprise sur deux critères : l'immobilité tonique (mesure de l'émotivité) et la motivation sociale.

Ces deux critères ont été choisis car ils présentent l'avantage de s'exprimer quel que soit le milieu d'élevage. Ils sont relativement faciles à mesurer et peuvent présenter un intérêt pratique puisqu'une faible émotivité devrait permettre d'avoir des animaux moins réactifs aux perturbations et qu'une forte motivation sociale pourrait entraîner une plus grande tolérance aux fortes densités. La caille a été choisie comme modèle en raison de son faible intervalle entre générations (jusqu'à 4 générations par an).

\section{a / Méthode de sélection}

La réaction d'immobilité tonique est obtenue en plaçant un animal sur le dos et en le maintenant pendant 10 secondes dans cette position. Une fois lâché, l'animal peut rester immobile pendant un temps qui varie de
Figure 11. Caille en immobilité tonique (cliché J.M. Faure).

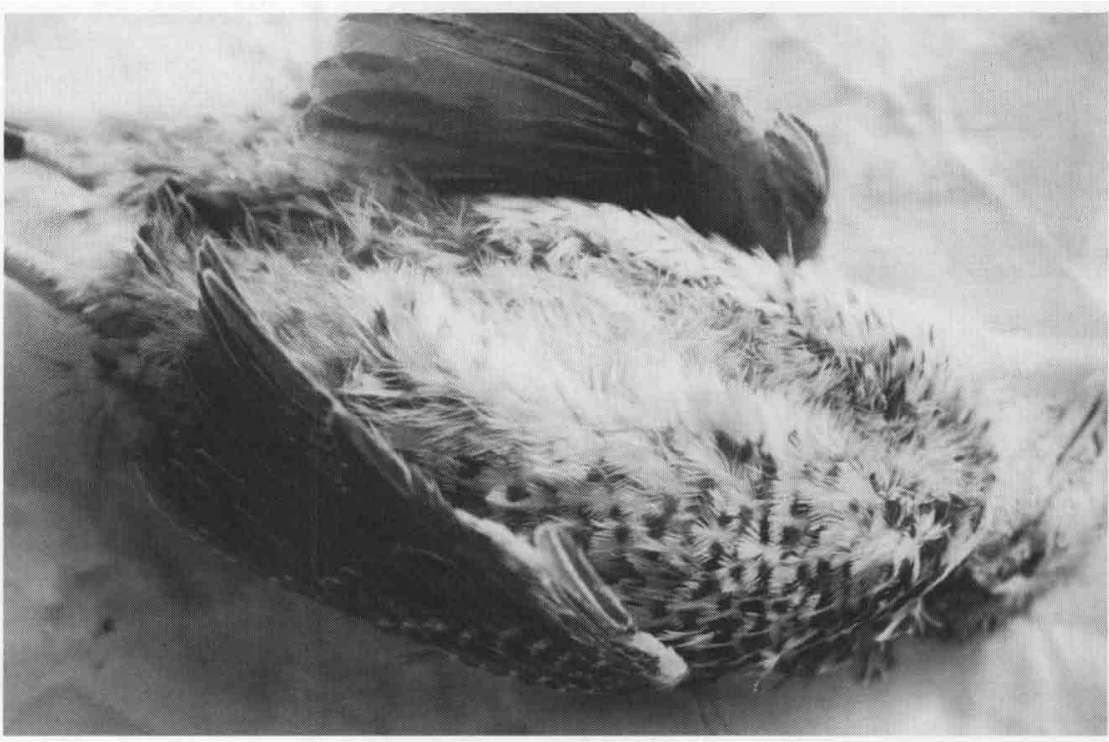

quelques secondes à quelques heures (figure 11). Dans le cas de notre sélection, nous interrompons le test après 5 minutes d'immobilité si l'animal ne s'est pas redressé et au moment où il se redresse si la durée d'immobilité est inférieure à 5 minutes.

La motivation sociale est estimée par la distance parcourue par un cailleteau sur un tapis roulant pour rejoindre un groupe de congénères (figure 12).

\section{b / Résultats de la sélection}

Les figures 13 et 14 montrent que dans les deux types de lignées la sélection a été très efficace et que les souches diffèrent largement pour les deux caractères sélectionnés. Nous disposons donc de 4 lignées sélectionnées (émotivité forte $(\mathrm{E}+)$ ou faible (E-), motivation sociale forte $\left(\mathrm{S}_{+}\right)$ou faible $(\mathrm{S}-)$ ) et de 2 lignées témoins. Ces différentes lignées ont été comparées dans de nombreux tests de réactivité comportementale et physiologique.
La caille, après avoir été maintenue pendant 10 secondes sur le dos, reste ensuite immobile pendant un temps dit "d'immobilité tonique" qui caractérise l'émotivité.

Figure 12. Schéma de l'appareil utilisé pour la mesure de la motivation sociale. Il est composé d'une petite cage, où est placé un groupe de cailleteaux stimuli, séparée de la grande cage par un grillage. Le cailleteau testé est placé dans la cage sur un tapis roulant qui se déplace si le cailleteau testé s'avance vers le groupe de congénères.

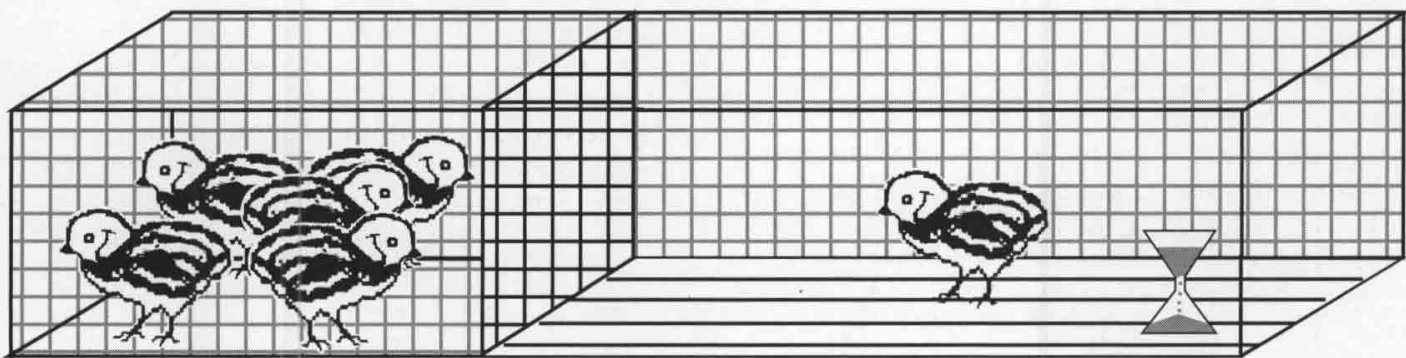

Groupe stimulus

(5 cailleteaux d'une souche témoin)
Déplacement du tapis roulant

S'approcher du groupe = sociabilité Mesure = "distance parcourue"
S'éloigner du groupe = non - sociabilité Mesure = "temps loin"


Figure 13. Résultats de la sélection de deux lignées divergentes de cailles sur leur durée d'immobilité tonique.

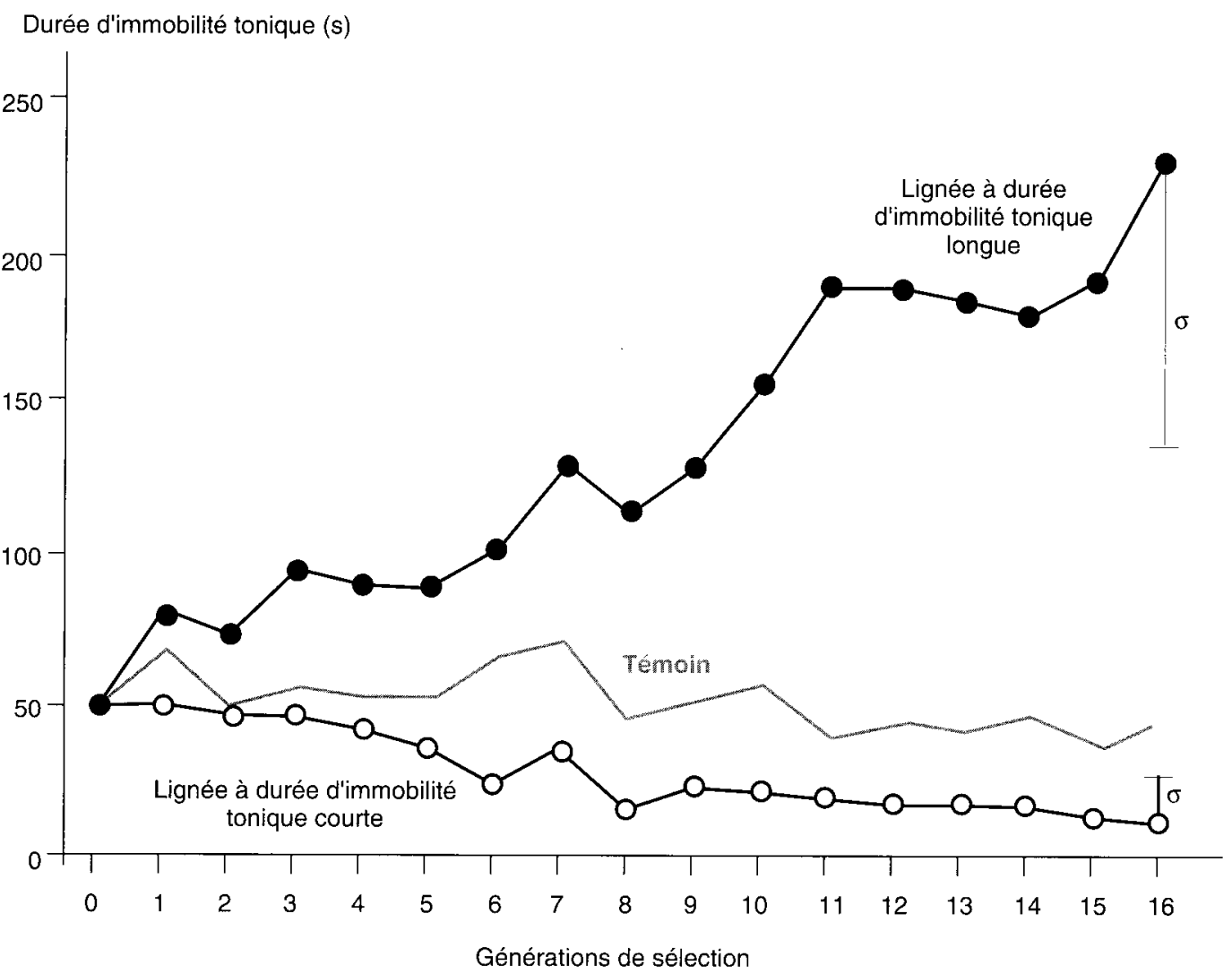

lignées de cailles sur des critères d'émotivité ou sur des critères de motivation sociale est très efficace.

Figure 14. Résultats de la sélection de deux lignées divergentes de cailles sur leur motivation sociale.

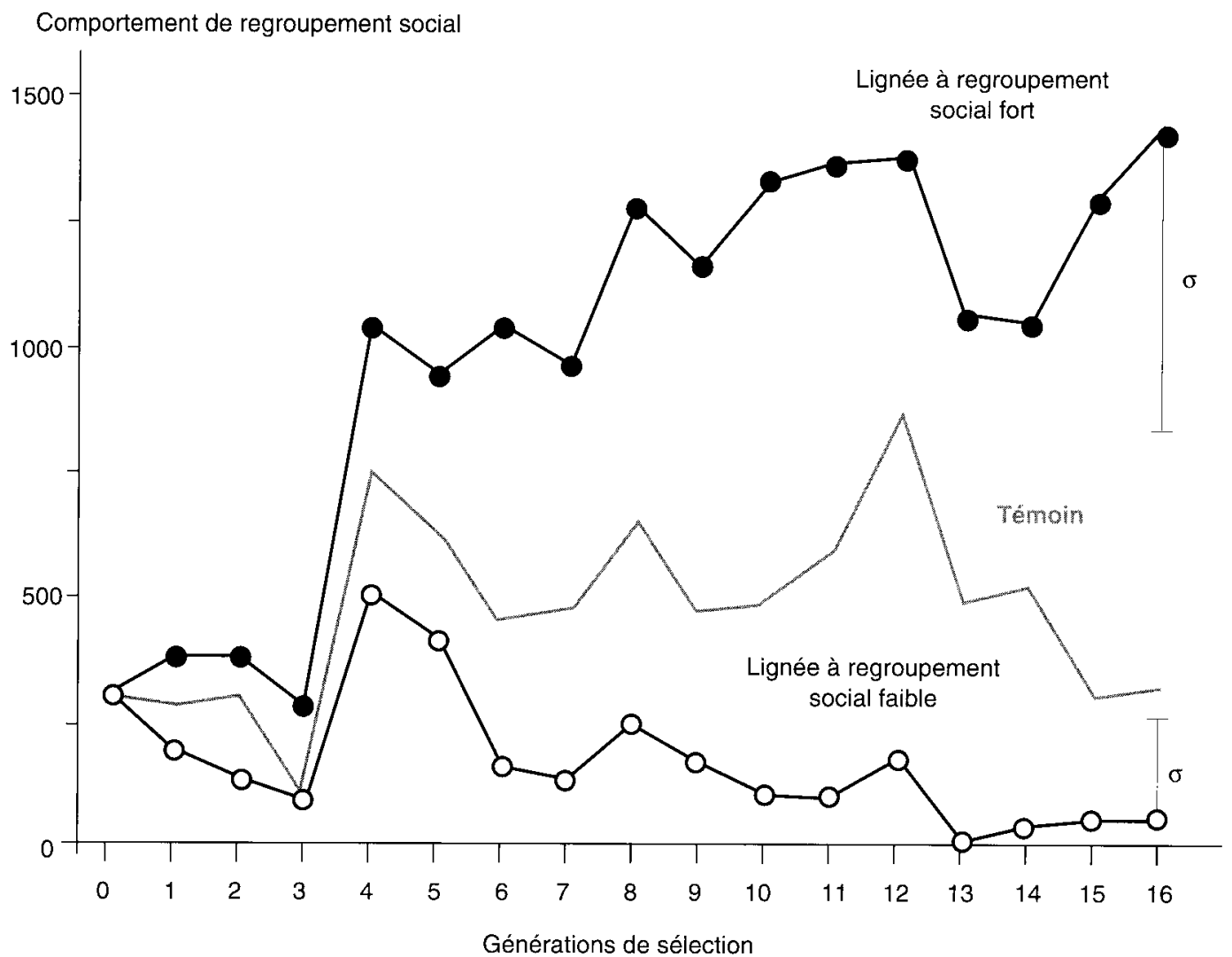


Les tests utilisés sont:

Le test d'open-field : l'animal est placé, seul, dans un environnement nouveau et son activité ainsi que les latences et fréquences des cris et des sauts sont notés pendant une période de 5 minutes.

Le test d'émergence : l'animal est placé, seul, dans une boîte obscure au centre d'un milieu éclairé. Le temps de sortie de la boîte est mesuré.

Le test de crainte : l'animal est immobilisé pendant 10 minutes (stress). La durée d'immobilité tonique est ensuite mesurée.

Stimulus inhabituel : un ballon de baudruche gonflé est introduit dans la cage où vivent les cailleteaux.

Enrichissement et manipulation : les animaux sont élevés dans un milieu enrichi (présence de nombreux objets colorés) ou non et sont manipulés 2 fois par jour ou non manipulés (plan factoriel).

Distances interindividuelles : elles ont été mesurées pendant un test d'open-field en paires, d'une durée de 10 minutes, par trajectométrie (analyse d'image vidéo).

Facilitation sociale du comportement alimentaire : elle est mesurée par la latence d'alimentation de cailleteaux placés devant un aliment nouveau en présence ou non d'un groupe de cailleteaux habitués à cet aliment et séparés des animaux testés par un grillage.

Test d'isolement : un cailleteau est isolé dans une cage identique à celle dans laquelle il a été élevé. Les résultats en sont résumés sur la figure 15.
On constate que :

- Les cailles émotives $(\mathrm{E}+)$ comparées aux cailles peu émotives (E-) :

. Sont moins actives en open-field

. Sortent plus lentement dans un test de timidité

Présentent une inhibition comportementale plus longue à la suite d'un stimulus auditif inhabituel

Sont les seules à présenter une augmentation du taux de corticostérone plasmatique à la suite d'un stimulus visuel inhabituel

. Sont moins réactives à la manipulation ou à l'enrichissement du milieu

- Les cailles à forte motivation sociale (S+) comparées aux cailles à faible motivation sociale (S-) :

. Sont plus actives, crient et sautent plus au cours d'un test d'open-field nères

Recherchent plus la proximité des congé-

Présentent des distances inter-individuelles plus faibles en test d'open-field par paire

Présentent une facilitation sociale du comportement alimentaire absent chez les cailles S-

Sont les seules à présenter une augmentation des cris, des sauts, de l'activité, du taux plasmatique de corticostérone et du rapport hétérophiles/ymphocytes au cours d'un test d'isolement.

L'ensemble de ces résultats est cohérent puisque chez les cailles sélectionnées pour leur émotivité, les différences portent sur des

Figure 15. Résultats synthétiques des comparaisons des souches sélectionnées.

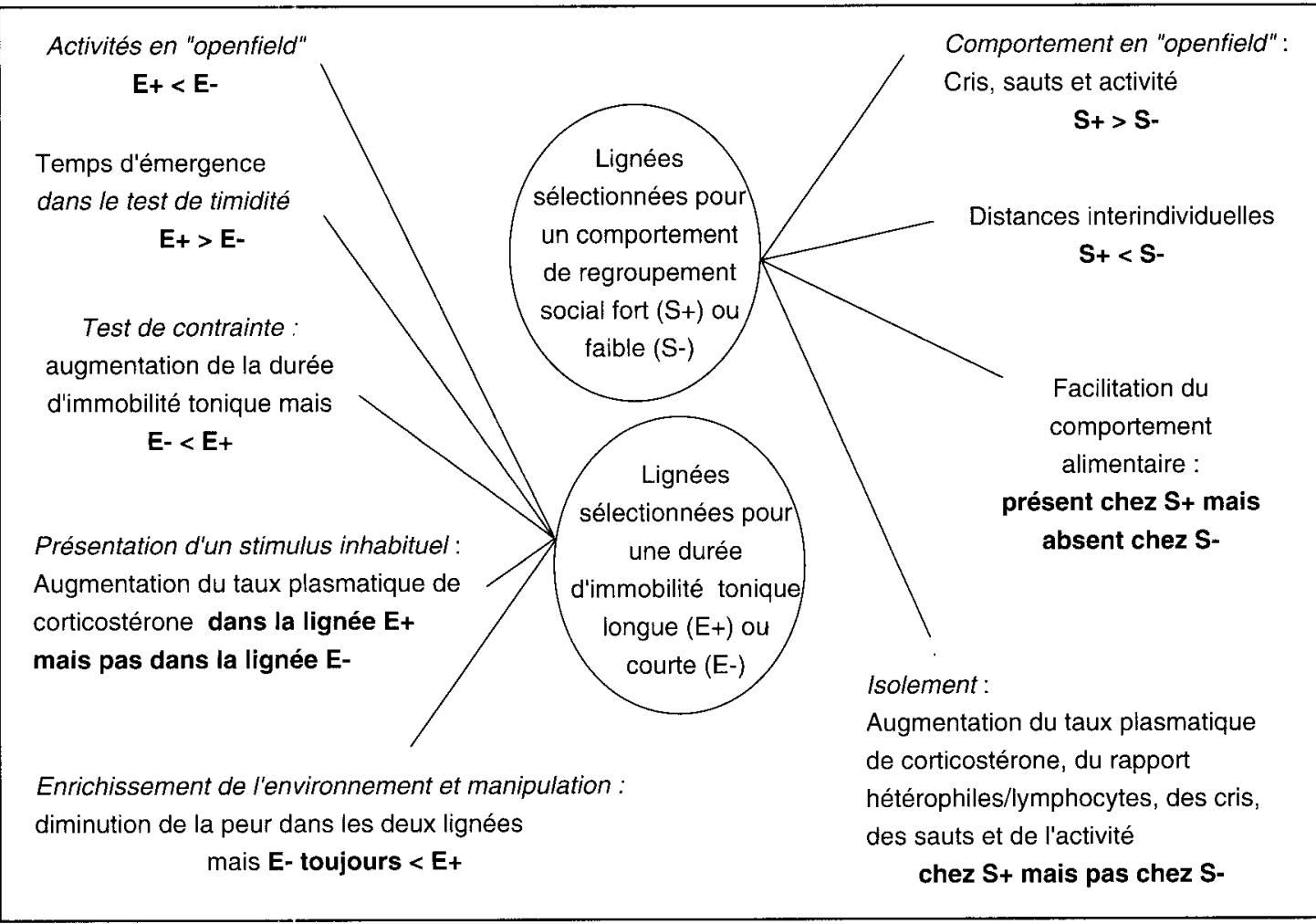


tests impliquant une forte nouveauté de la situation de test alors que chez les cailles sélectionnées pour leur motivation sociale c'est l'aspect social de la situation qui permet de mettre en évidence des différences entre les deux souches.

\section{4 / Applications possibles}

Trois types d'applications sont possibles avec une approche visant à modifier l'animal pour l'adapter à son environnement :

- Une meilleure connaissance de l'ontogenèse des animaux peut permettre de modifier l'environnement des juvéniles afin d'adapter leur comportement aux conditions qu'ils rencontreront à l'âge adulte. Le travail d'Appleby et al (1988) est une bonne illustration de cette approche puisque cet auteur a montré que dans le cas de pondeuses élevées au sol et devant se percher pour accéder aux nids, la présence de perchoirs dès l'âge de 4 semaines permet aux poules adultes d'avoir un taux de ponte au sol très inférieur à celui d'animaux n'ayant eu accès à des perchoirs qu'à un âge plus tardif.

- Une meilleure connaissance des caractéristiques des lignées existantes peut bien sûr permettre de choisir celles qui sont le mieux adaptées à un type de production.

- La mise au point de tests de comportement suffisamment courts pour être utilisables en sélection, ainsi que la démonstration des effets favorables de ce type de sélection doit enfin permettre d'offrir aux sélectionneurs des moyens d'améliorer l'adaptabilité de leurs lignées.

\section{Conclusions}

Actuellement, les éleveurs privilégient l'élevage des poules en cage alors que les protecteurs des animaux y sont farouchement opposés. La cage est surtout critiquée pour le manque d'espace disponible et l'absence de litière. Ces deux facteurs ne semblent cependant pas essentiels pour la poule. La cage présente, par contre, l'avantage de réduire les risques de cannibalisme (et donc de supprimer la nécessité du débecquage) ainsi que les risques sanitaires. Elle présente, par contre, l'inconvénient de restreindre la liberté des animaux et leurs possibilités d'exprimer l'ensemble de leurs comportements.

Les systèmes alternatifs (élevage sur litière ou en volière avec ou sans parcours herbeux) présentent les caractéristiques inverses : risques de cannibalisme et risques sanitaires importants, mais plus grande liberté des animaux. Il y a donc opposition entre d'une part, un milieu très contrôlé par l'éleveur et, d'autre part, un milieu offrant des possibilités de contrôles plus limitées tant sur le plan sanitaire que climatique ou comportemental.

Les deux types d'approches présentés ici nous ont permis de montrer que l'on peut d'une part, améliorer les conditions d'élevage, à condition de connaître les besoins des animaux et, d'autre part, améliorer leur adaptation aux conditions qui leur sont imposées. Ces deux types d'approche utilisés de manière conjointe devraient permettre d'arriver à un compromis entre les demandes des éleveurs et des protecteurs des animaux si les deux parties acceptent de placer le débat sur un plan rationnel.

\section{Remerciements}

Tous les résultats expérimentaux cités sans référence (les références complètes peuvent être obtenues auprès des auteurs) ont été obtenus à la Station de Recherches Avicoles, en grande partie au cours du travail de thèse de H. Lagadic et de F. Launay qui ont respectivement bénéficié d'une bourse de la région Bretagne et d'une bourse de la région Centre. M. Marché et J.M. Meslier doivent aussi être remerciés pour leur assistance technique.

\section{Références bibliographiques}

Appleby M.C., Duncan I.J.H., Mc Rae H.E., 1988. Perching and floor laying by domestic hens : experimental results and their commercial application. $\mathrm{Br}$. Poult. Sci., 29, 351-357.

Boissy A., Bouissou M.F., 1988. Effect of early handling on heifer's subsequent reactivity to humans and to unfamiliar situations. Appl. Anim. Behav. Sci., 20, 259-273.

Dawkins M., 1983. Battery hens name their price; consumer demand theory and the measurement of ethological « needs ». Anim. Behav., 31, 1195-1205.
Dawkins M., Beardsley T., 1986. Reinforcing properties of access to litter in hens. Appl. Anim. Behav. Sci., 15, 351-364.

Faure J.M., Jones R.B., 1982. Effect of age, access and time of day on perching behaviour in the domestic fowl. Appl. Anim. Ethol., 8, 357-364.

Jones R.B., Faure J.M., 1981. The effect of regular handling on fear responses in the domestic chick. Behav. Process., 6, 135-143.

Nicol C. J., 1987. Effect of cage height and area on the behaviour of hens housed in battery cages. Br. Poult. Sci., 28, 327-335. 


\begin{abstract}
Behaviour and welfare in domestic birds.

The notion of animal welfare has two aspects: the perception by the public (consumers) of the conditions under which domestic animals are housed and the perception by the animals, themselves, of the conditions under which they are housed.

Only the second of these two aspects can be addressed by behavioural studies. Two approaches to the improvement of animal welfare exist : adaptation of the environment (housing system) to the animal and adaptation of the animal to the environment.

A pre-requisite of the first of these approaches is a clear understanding of the behavioural needs of the animals. Even in the domestic chicken, the acquisition of the information necessary for such an understanding is still incomplete. This point is

illustrated using data from experiments with four different methodological approaches : comparison of the behaviour of animals in different environments, operant conditioning, choice tests and the induction of motivational conflict.

The adaptation of an animal to its environment can be effected in two ways : ontogenetic and genetic. Data from studies using each of these approaches are given.

It is concluded that animal welfare is most likely to be improved by the combination of environmental modification and genetic adaptation of domestic animals to modern husbandry conditions.

FAURE J.M., MILLS A.D., 1995. Bien-être et comportement chez les oiseaux domestiques. INRA Prod. Anim., 8 (1), 57-67.
\end{abstract}

